\title{
Epidemiology and Clinical Outcomes of Inflammatory Bowel Disease: A Hospital-Based Study in Central Taiwan
}

\author{
Jen-Wei Chou $\mathbb{D}^{1,2,3,4}$ Hsiang-Chun Lai ${ }^{1},{ }^{5}$ Chia-Hsi Chang, ${ }^{2}$ Ken-Sheng Cheng, ${ }^{1,2}$ \\ Chun-Lung Feng, ${ }^{2}$ and Tsung-Wei Chen ${ }^{6}$ \\ ${ }^{1}$ School of Medicine, China Medical University, Taichung, Taiwan \\ ${ }^{2}$ Division of Gastroenterology and Hepatology, Department of Internal Medicine, China Medical University Hospital, \\ Taichung, Taiwan \\ ${ }^{3}$ Taiwan Society of Inflammatory Bowel Disease, Taichung, Taiwan \\ ${ }^{4}$ Taiwan Association for the Study of Small Intestinal Diseases, Taoyuan, Taiwan \\ ${ }^{5}$ Department of Chinese Medicine, China Medical University Hospital, Taichung, Taiwan \\ ${ }^{6}$ Department of Pathology, China Medical University Hospital, Taichung, Taiwan \\ Correspondence should be addressed to Hsiang-Chun Lai; u9702451@cmu.edu.tw
}

Received 13 February 2019; Revised 5 May 2019; Accepted 15 May 2019; Published 13 June 2019

Academic Editor: Paolo Gionchetti

Copyright (C) 2019 Jen-Wei Chou et al. This is an open access article distributed under the Creative Commons Attribution License, which permits unrestricted use, distribution, and reproduction in any medium, provided the original work is properly cited.

\begin{abstract}
The incidence and prevalence of inflammatory bowel disease (IBD) are low but increasing in Taiwan. We aimed to investigate the epidemiology and clinical outcomes of IBD in central Taiwan. We retrospectively analyzed patients with IBD diagnosed at our hospital between January 2000 and September 2018. The diagnostic criteria were based on endoscopic and pathologic findings. Clinical characteristics, treatment regimens, and treatment outcomes were analyzed. A total of 190 patients with IBD were enrolled ( 80 with Crohn's disease (CD) and 110 with ulcerative colitis (UC)). The mean age at diagnosis was 38.4 years (CD: 36 years, UC: 40 years). Male patients accounted for the majority of patients (71.1\%). The male-to-female ratio was $3: 1$ for CD and 2.1:1 for UC. Current and ever smokers accounted for 30.5\% of all patients. Only $4.2 \%$ of patients had a family history of IBD. Extraintestinal manifestations (EIMs) were reported in $7.9 \%$, and colorectal cancers (CRCs) were reported in $2.1 \%$ of all patients. In patients with $\mathrm{CD}$, the ileal type was the most common disease phenotype $(57.5 \%)$, and the stricturing type was the most common disease behavior (60.0\%). In patients with UC, left-sided colitis was the predominant disease extent (42.7\%). The seroprevalence of hepatitis B virus (HBV) was $13.3 \%$. The incidence of perinuclear anti-neutrophil cytoplasmic antibody (p-ANCA) in patients with UC was $22 \%$. 5-Aminosalicylic acids were the preferred treatment for UC, whereas corticosteroids, immunomodulators, and biologic agents were preferred for CD. In patients with CD, the bowel resection rate was $38.8 \%$, and the incidence of hip avascular necrosis was 3.8\%. In Taiwan, patients with IBD showed a male predominance, lack of familial clustering, a higher prevalence of HBV infection, and a lower prevalence of p-ANCA, EIMs, and CRC. Moreover, a higher incidence of the ileal type with poor outcomes of CD and left-sided predominance in UC were found.
\end{abstract}

\section{Introduction}

Inflammatory bowel disease (IBD), which includes Crohn's disease (CD) and ulcerative colitis (UC), is a chronic relapsing and remitting disease. Although there have been numerous epidemiologic studies on IBD, the etiology of this disease remains unclear. UC mainly affects the colon and rectum, whereas CD can affect the entire gastrointestinal (GI) tract. IBD was believed to be a Western disease, as it is most prevalent in North America, Europe, and Oceania. The prevalence of IBD in the Western world is about 50-200 per 100,000 persons for CD and 120-200 per 100,000 persons for UC [1]. However, the incidence and prevalence rates of IBD have shown an increasing trend in Asia and Africa [2]. Therefore, IBD has become a global disease in the 21st century with a large socioeconomic burden $[2,3]$.

The incidence and prevalence of IBD are low in Taiwan compared with Western countries, but they have shown an 
TABLE 1: Clinical characteristics of patients with inflammatory bowel disease.

\begin{tabular}{|c|c|c|c|c|}
\hline Characteristics & $\begin{array}{l}\text { Total IBD } \\
(N=190)\end{array}$ & $\begin{array}{c}\mathrm{CD} \\
(n=80)\end{array}$ & $\begin{array}{c}\mathrm{UC} \\
(n=110)\end{array}$ & $p$ value \\
\hline Male sex, $n(\%)$ & $135(71.1)$ & $60(75.0)$ & $75(68.2)$ & 0.306 \\
\hline Mean age at diagnosis (years) $( \pm S D)$ & $38.4 \pm 15.9$ & $36.0 \pm 17.4$ & $40.1 \pm 14.6$ & 0.080 \\
\hline Cigarette smoking & & & & 0.002 \\
\hline Nonsmoking, $n(\%)$ & $132(69.5)$ & $50(62.5)$ & $82(74.5)$ & \\
\hline Ever smoking, $n(\%)$ & $32(16.8)$ & $11(13.8)$ & $21(19.1)$ & \\
\hline Current smoking, $n(\%)$ & $26(13.7)$ & $19(23.7)$ & $7(6.4)$ & \\
\hline Alcohol consumption & & & & 0.194 \\
\hline Nondrinking, $n(\%)$ & $156(82.1)$ & $70(87.5)$ & $86(78.2)$ & \\
\hline Ever drinking, $n(\%)$ & $10(5.3)$ & $3(3.8)$ & $7(6.4)$ & \\
\hline Current drinking, $n(\%)$ & $24(12.6)$ & $7(8.7)$ & $17(15.4)$ & \\
\hline Family history of IBD, $n(\%)$ & $8(4.2)$ & $2(2.5)$ & $6(5.5)$ & 0.317 \\
\hline Extraintestinal manifestations, $n(\%)$ & $15(7.9)$ & $7(8.8)$ & $8(7.3)$ & 0.709 \\
\hline Colorectal cancers, $n(\%)$ & $4(2.1)$ & $1(1.2)$ & $3(2.7)$ & 0.484 \\
\hline Fistula formation, $n(\%)$ & & $22(27.5)$ & & \\
\hline Positivity of $\mathrm{HBsAg}^{\wedge}, n(\%)$ & $21(13.3)$ & $9(11.3)$ & $12(15.4)$ & 0.444 \\
\hline Positivity of anti-HCV $\mathrm{Ab}^{\wedge}, n(\%)$ & $0(0)$ & $0(0)$ & $0(0)$ & \\
\hline Positivity of p-ANCA*, $n(\%)$ & & & $14(22.2)$ & \\
\hline Positivity of c-ANCA ${ }^{*}, n(\%)$ & & & $1(1.6)$ & \\
\hline
\end{tabular}

Abbreviations: anti-HCV Ab: anti-hepatitis C virus antibody; c-ANCA: cytoplasmic anti-neutrophil cytoplasmic antibody; CD: Crohn's disease; HBsAg: hepatitis B surface antigen; IBD: inflammatory bowel disease; p-ANCA: perinuclear anti-neutrophil cytoplasmic antibody; UC: ulcerative colitis. ${ }^{\wedge}$ There were 158 patients after excluding those with missing data. *There were 63 patients after excluding those with missing data.

increasing trend in the recent 2 decades [3, 4]. This trend might be attributed to the Westernized lifestyle [4]. In East Asia, there are unique regional environmental factors, such as a higher prevalence of hepatitis $\mathrm{B}$ virus (HBV) and tuberculosis infection, which cause some differences in the characteristics of patients with IBD. Moreover, it has been reported that UC has milder severity in Asia [5]. In the literature on IBD in Taiwan, most of the epidemiologic studies are population-based studies that used data from the Taiwan National Health Insurance Database, which tend to underestimate the true prevalence and incidence. However, hospitalbased studies on IBD are scarce. Population-based studies may have some errors because of incorrect coding. Moreover, such studies may lack details of clinical characteristics and treatment programs. In our present study, we aimed to investigate the clinical characteristics and treatments of patients with IBD through a hospital-based study in central Taiwan.

\section{Materials and Methods}

2.1. Study Population. We searched the database of chart records of China Medical University Hospital (CMUH), a tertiary medical center in central Taiwan, from January 2000 to September 2018. We also searched the International Classification of Disease (2001 version) for disease coding, according to which UC is coded as 556.XX and CD as 555.XX in our chart records. We enrolled patients with a regular follow-up for at least 3 months after the original diagnosis of IBD. All data were completely reviewed by Dr. Chou, a gastroenterologist at $\mathrm{CMUH}$. The diagnostic criteria for
IBD were based on a combination of clinical presentations, endoscopic features, and pathologic findings (infectious and malignant etiologies were excluded). The follow-up duration of our patients was from the time of diagnosis or the first hospital visit for treatment to the last record in our chart.

We collected clinical data including sex, age at diagnosis, alcohol consumption/smoking habits, family history, disease phenotype, and behavior at diagnosis according to the Montreal classification, extraintestinal manifestations (EIMs), presence of fistula, colorectal cancers (CRCs), avascular necrosis (AVN) of the hips, hepatitis $B$ virus (HBV)/hepatitis C virus (HCV) infection status, and antineutrophil cytoplasmic antibody (ANCA) status. The clinical course of the patients was recorded and analyzed. We also collected and analyzed the treatment regimens and bowel resection rates in our patients with IBD.

2.2. Statistical Methods. For statistical methods, we used Pearson's chi-square test for categorical variables and Student's $t$-test for continuous variables.

2.3. Ethical Considerations. This study was approved by the institutional review board of the research ethics committee of CMUH in central Taiwan (CMUH107-REC1-139).

\section{Results}

A total of 190 patients with IBD were enrolled in our present study. The clinical characteristics of all patients are shown in Table 1. There were 80 patients with CD and 110 patients 
with UC. The mean age at diagnosis of all patients was $38.4 \pm 15.9$ years (range: $9-85$ years). Although the mean age at diagnosis of patients with $\mathrm{CD}$ was lower than that of patients with UC, there was no statistical significance between these 2 groups (CD: 36 years vs. UC: 40 years, $p=0.080$ ). With respect to the sex distribution, male patients accounted for the majority $(71.1 \%)$ of all patients. The male-to-female ratio was $3: 1$ in the CD group and 2.1:1 in the UC group. The proportion of male patients showed no significant difference between the CD and UC groups (CD: $75.0 \%$ vs. UC: $68.2 \%, p=0.306$ ). With respect to the smoking status, the proportion of current smokers, ever smokers, and nonsmokers was $13.7 \%, 16.8 \%$, and $69.5 \%$, respectively. There was a significant difference in the proportion of patients with a smoking habit between the CD and UC groups $(p=0.002)$. Concerning alcohol consumption, the incidence of current drinking, social drinking, ever drinking, and nondrinking was $1.6 \%, 11.1 \%, 5.3 \%$, and $82.1 \%$, respectively. There was no statistical difference between the CD and UC groups in the habit of alcohol consumption $(p=0.194)$. In the investigation of family history of IBD in all patients, we found only $4.2 \%$ with a family history, with no significant difference between the CD and UC groups (CD: $2.5 \%$ vs. UC: $5.5 \%$, $p=0.317)$. In the analysis of EIMs, the incidence of EIM was $7.9 \%$ in all patients with IBD. There was no significant difference in the incidence of EIM between the CD and UC groups (CD: $8.8 \%$ vs. UC: $7.3 \%, p=0.709$ ). The incidence of CRC in all patients was $2.1 \%$. There was no significant difference between patients with $\mathrm{CD}$ and those with UC (CD: $1.2 \%$ vs. UC: $2.7 \%, p=0.484$ ). There were some missing data in laboratory tests, including $\mathrm{HBV} / \mathrm{HCV}$ infection status and serum ANCAs. We analyzed these variables by excluding missing data. The incidence of $\mathrm{HBV}$ infection was $13.3 \%$ in 158 patients with IBD with available data. However, there was no significant difference between the $\mathrm{CD}$ and UC groups (CD: $11.3 \%$ vs. UC: $15.4 \%, p=0.444$ ). The incidence of perinuclear ANCA (p-ANCA) was $22 \%$ and that of cytoplasmic ANCA (c-ANCA) was $1.6 \%$ in 63 patients with UC with available data.

The Montreal classifications of all patients with IBD in our study are summarized in Table 2 . With respect to the disease phenotype of $\mathrm{CD}$, we found $57.5 \%, 7.5 \%, 33.8 \%$, and $1.3 \%$ of cases to be located in L1 (ileum), L2 (colon), L3 (ileo-colon), and L4 (upper GI tract) at diagnosis, respectively. Concerning the disease behavior of CD, B1 (nonstricturing and nonpenetrating type), B2 (stricturing type), and B3 (penetrating type) were observed $18.8 \%, 60.0 \%$, and $21.3 \%$, respectively. The ileal type and stricturing type accounted for the majority of our CD cases. In the disease extension of UC, we found that the incidence of E1, E2, and E3 at diagnosis was $18.2 \%, 42.7 \%$, and $39.1 \%$, respectively. Left-sided UC accounted for the majority of UC cases in our study. Of the patients with CD, 17 (21.3\%) had perianal involvement and $22(27.5 \%)$ were complicated with fistula formation.

The treatment regimens, bowel resection rates, and hip AVN incidence in our patients with IBD are summarized in Table 3. The treatment regimens for IBD in our present study included 5-aminosalicylic acids (5-ASAs), corticosteroids,
TABLE 2: Montreal classification of patients with inflammatory bowel disease.

\begin{tabular}{lcc}
\hline & $\begin{array}{c}\text { CD } \\
(n=80)\end{array}$ & $\begin{array}{c}\text { UC } \\
(n=110)\end{array}$ \\
\hline UC disease extent & & \\
E1: proctitis, $n$ (\%) & & $47(42.7)$ \\
E2: left-sided colitis, $n(\%)$ & $43(39.1)$ \\
E3: extensive colitis, $n(\%)$ & & \\
CD disease phenotype & & \\
L1: ileum, $n(\%)$ & $66(57.5)$ & \\
L2: colon, $n(\%)$ & $27(7.5)$ & \\
L3: ileo-colon, $n(\%)$ & $1(1.3)$ & \\
L4: UGI tract, $n(\%)$ & & \\
CD disease behavior & $15(18.8)$ & \\
B1: nonstricturing, nonpenetrating ${ }^{\#}$, & \\
$n$ (\%) & $48(60.0)$ & \\
B2: stricturing ${ }^{\#}, n(\%)$ & $17(21.3)$ & \\
B3: penetrating ${ }^{\#}, n(\%)$ & $17(21.3)$ & \\
P: perianal involvement, $n(\%)$ & & \\
\hline
\end{tabular}

Abbreviations: CD: Crohn's disease; UC: ulcerative colitis; UGI: upper gastrointestinal. "Includes cases with perianal involvement.

immunomodulators, and biologic agents. Our data showed that 5-ASAs were more preferred in patients with UC than in those with CD. There was a statistical difference between the UC and CD groups (UC: $99.1 \%$ vs. CD: $93.8 \%$, $p=0.038$ ). Moreover, the uses of corticosteroids (CD: $80.0 \%$ vs. UC: $61.8 \%, p=0.007)$, immunomodulators (CD: $63.8 \%$ vs. UC: $27.3 \%, p<0.001$ ), and biologic agents (CD: $85.0 \%$ vs. UC: $42.7 \%, p<0.001$ ) all showed a statistically significant difference between patients with $\mathrm{CD}$ and those with UC. In our study, the bowel resection rate in all patients with IBD was $18.4 \%$. Patients with CD showed a significantly higher bowel resection rate than those with UC (CD: $38.8 \%$ vs. UC: $3.6 \%, p<0.001$ ). The prevalence of hip AVN in all patients was $3.2 \%$. Although patients with CD showed a trend of having more AVN complications than patients with UC, there was no significant difference between the groups (CD: $3.8 \%$ vs. UC: $2.7 \%, p=0.484$ ).

\section{Discussion}

IBD used to be known as a Western disease because of its higher prevalence in North America, Europe, and Oceania [3]. Recent reports demonstrated that the incidence and prevalence rates of IBD in Asia have been increasing [6]. Wei et al. reported an epidemiologic study of IBD from 1998 to 2008 in Taiwan. They found that the prevalence of CD increased from $0.19 / 100,000$ to $1.78 / 100,000$ and that of UC increased from $0.61 / 100,000$ to $7.62 / 100,000$ [7]. These results may be attributed to urbanization, lifestyle changes, diet, and advanced diagnostic methods such as enteroscopy [8]. Thus, IBD has become an important issue in Asian people.

With respect to the prevalence of IBD types, UC seemed to be more prevalent than CD $(202 / 100,000$ vs. $146 / 100,000)$ 
TABLE 3: Treatment regimens and clinical outcomes of patients with inflammatory bowel disease.

\begin{tabular}{|c|c|c|c|c|}
\hline Treatment regimens and outcomes & $\begin{array}{l}\text { Total IBD } \\
(N=190)\end{array}$ & $\begin{array}{c}\text { CD } \\
(n=80)\end{array}$ & $\begin{array}{c}\mathrm{UC} \\
(n=110)\end{array}$ & $p$ value \\
\hline 5-ASAs, $n(\%)$ & $184(96.8)$ & $75(93.8)$ & $109(99.1)$ & 0.038 \\
\hline Corticosteroids, $n(\%)$ & $132(69.5)$ & $64(80.0)$ & $68(61.8)$ & 0.007 \\
\hline Immunomodulators, $n(\%)$ & $81(42.6)$ & $51(63.8)$ & $30(27.3)$ & $<0.001$ \\
\hline Biologic agents, $n(\%)$ & $115(60.5)$ & $68(85.0)$ & $47(42.7)$ & $<0.001$ \\
\hline Bowel resection, $n(\%)$ & $35(18.4)$ & $31(38.8)$ & $4(3.6)$ & $<0.001$ \\
\hline Avascular necrosis of the hips, $n(\%)$ & $6(3.2)$ & $3(3.8)$ & $3(2.7)$ & 0.484 \\
\hline
\end{tabular}

Abbreviations: 5-ASAs: 5-aminosalicylic acids; CD: Crohn's disease; IBD: inflammatory bowel disease; UC: ulcerative colitis.

[9]. In our study, we found comparable results for the UC and CD ratios. Several cohort studies have indicated a female predominance in CD and a male predominance in UC [1]. The sex distribution of IBD has been consistently significantly different worldwide. IBD is a male-predominant disease in Asia, according to previous studies. In our present study, we also observed a male predominance $(75.0 \%$ in CD and $68.2 \%$ in UC). These ratios were higher than those reported in previous Asia-Pacific studies, in which male patients constituted $59.6 \%$ of CD cases and $57.7 \%$ of UC cases [8]. However, a Korean study reported that male patients constituted $73 \%$ of CD cases, which is comparable to our data [6]. With respect to the age at diagnosis of IBD, the peak age at onset for CD was between 20 and 30 years and that for UC was between 30 and 40 years [10]. Our patients tended to be diagnosed at an older age with $\mathrm{CD}$ but at a younger age with UC (CD: 36.0 years, UC: 40.1 years) compared with the patients in another study (CD: 33 years, UC: 43 years) [8]. A consistent trend was observed that patients with $\mathrm{CD}$ had a younger age at diagnosis than did patients with UC. Nevertheless, there was no significant difference between the ages of patients with CD and those with UC. The reason may be the small number of our patients. Smoking is one of the known environmental protective factors for UC, but it is a risk factor for CD [11]. The higher prevalence rate of cigarette smoking also had an association with lower income levels in America, Europe, and Asia [12]. Birrenbach and Bocker reported a wide range of prevalence rates of cigarette use in patients with IBD (CD: 39-72\%, UC: $11-23 \%)$ and found that nonsmokers were more common among patients with UC than among those with CD [13]. Our present study found that among patients with $\mathrm{CD}, 23.8 \%$ were current smokers and $13.8 \%$ were ever smokers, and these percentages were higher than those reported in another Asia-Pacific study (11.6\% current smokers and $11.3 \%$ ever smokers) [8]. Men have a greater tendency to smoke than women in Asia [14]. This high smoking rate in patients with CD might have partially contributed to the higher proportion of male patients in our study; however, more studies are needed to prove this assumption. In our study, there were $6.4 \%$ current smokers and $19.1 \%$ ever smokers among patients with UC, and these percentages were comparable to those reported in a previous study [8]. Some Asian cohort studies demonstrated that smoking was a protective factor against UC [15]. In the English literature, we found fewer data on alcohol consump- tion than on tobacco usage among patients with IBD. The incidence of alcohol consumption in our results was lower than that in previous studies (39.5\% ever drinking and $46.9 \%$ binge drinking) $[16,17]$. Additionally, evidence showing that alcohol consumption exacerbates IBD remains equivocal. Some studies reported that alcohol is a sulfur and sulfate producer, thus increasing the concentration of fecal hydrogen sulfide (an agent toxic to colonocytes) [18-20]. Patients with IBD in Asia tend to have a lower positive family history rate $(0.0-3.4 \%)$ than those in Western countries $(10-25 \%)[2,21,22]$. The rate of positive family history of IBD $(4.2 \%)$ in our study was consistent with that in other Asian reports. However, recent Asian studies demonstrated an increased positive family history rate of IBD patients, which might be attributable to a higher recognition of IBD [23].

With respect to the disease phenotype of $\mathrm{CD}$ according to the Montreal classification, equal proportions of phenotypes were reported in patients with $\mathrm{CD}$, whereas some studies reported that the colonic type was predominant in Western people. Prideaux et al. demonstrated that the ileo-colonic type was the most common type of CD in Asia (about $30-50 \%$ ), followed by the ileal and colonic types [2]. $\mathrm{Ng}$ et al. reported more equal distributions of the ileal, colonic, and ileo-colonic types (31.7\%, 24.4\%, and 43.9\%, respectively) and that the incidence of the upper GI type was $6 \%$, consistent with previously reported hospital-based data in Taiwan. In our study, we identified that the most common phenotype was the ileal type, followed by the ileocolonic, colonic, and upper GI types. Concerning the disease behavior of $\mathrm{CD}$, the nonstricturing and nonpenetrating type was the most common type in Western reports (62-81\%) compared with Asian reports (40-69\%) [2, 8, 24]. In our study, we found that the most dominant disease behavior was the stricturing type, followed by the penetrating type and then the nonstricturing and nonpenetrating type. Our results showed that patients with $C D$ in Taiwan had higher ratios of the stricturing and penetrating types at diagnosis, with poor prognostic outcomes. The first reason may be the delay in diagnosis owing to the lack of awareness of CD. The second reason is that ileal-type $\mathrm{CD}$ is difficult to diagnose because of the need for small-bowel endoscopy, which is a challenging procedure for most endoscopists in Taiwan. In the literature, the incidence of perianal involvement ranges from $15.6 \%$ to $36.7 \%$ in patients with $\mathrm{CD}[2,8]$. We reported an incidence of $21.3 \%$, which was comparable to 
that in a previous report in Taiwan [24]. Schwartz et al. demonstrated that $20-40 \%$ of patients with CD developed a fistula (mostly perianal fistula followed by enteroenteric fistula) and the cumulative risk of any fistula formation was $50 \%$ after 20 years [25]. In our study, 27.5\% of all patients had a fistula (mainly anal fistula formation), comparable to the report by Schwartz et al. With respect to the disease extent of UC, large variations have been reported worldwide. Western reports showed proctitis in 30-60\%, left-sided colitis in $16-40 \%$, and extensive colitis in $18-35 \%$ of patients with UC. However, Asia-Pacific reports demonstrated equal distributions among these 3 types $(32.6 \%, 34.9 \%$, and $36.2 \%$, respectively) $[2,8]$. In the present study, the most common type was left-sided UC, followed by extensive colitis and proctitis, which was comparable to a study in Japan involving 10,390 patients [26]. The prevalence of EIMs in patients with IBD was estimated to be $25-50 \%$ in Western countries, and EIMs were more common in CD than in UC [27]. Prideaux et al. reported that $3.7-24 \%$ of patients with IBD in East Asia had EIMs, and the prevalence was higher in India [2]. Wei et al. reported that EIMs occurred in 1\% of CD cases and in $4.5 \%$ of UC cases in their hospital-based study in Taiwan $[5,24]$. Our study found that $7.9 \%$ of patients with IBD had EIMs, which was similar to that in previous Asian reports. Concerning the prevalence of CRCs in patients with UC, it has been reported as $3-5 \%$ in Western countries and $0.0-2.2 \%$ in Asia [2]. Only a few studies on CRCs associated with CD have been reported in the literature, which demonstrated an increased risk in patients with colonic-type CD but without significance [28]. Eaden et al. conducted a meta-analysis study excluding Asian countries and found that the cumulative rate was $2 \%, 8 \%$, and $18 \%$ in the first, second, and third decades, respectively [29]. Our present study showed that the incidence of CRCs in patients with IBD was $2.1 \%$, which is comparable to that reported in previous Asian studies. This low incidence of CRCs might be related to the relatively short follow-up duration in many Asian studies. Thereby, we intend to continue following up our patients.

Because Asian countries are an endemic area for HBV infection, they have a higher seroprevalence of hepatitis B surface antigen (HBsAg) than North America and Europe do [30]. The seroprevalence of HBsAg in patients with IBD varies worldwide. In a report from China, the seroprevalence of $\mathrm{HBsAg}$ was $5.29 \%$ in patients with $\mathrm{CD}$ and $5.68 \%$ in patients with UC [31]. This study concluded that there was a higher seroprevalence of HBsAg in patients with IBD than in healthy controls. The Taiwanese government implemented a universal HBV mass vaccination program for infants in 1984. Thus, the seroprevalence of HBsAg in the general population was reported to be $15-20 \%$ before the HBV vaccination implementation and 13.7\% in 2007 (after HBV vaccination implementation) $[32,33]$. In young adults receiving HBV vaccination in Taiwan, the seroprevalence of HBsAg was reported to be $0.9 \%$ in 2011 and $1.1 \%$ in 2012 $[34,35]$. Our present study showed that the seroprevalence of $\mathrm{HBsAg}$ was $13.3 \%$ in 158 patients with IBD with available data (CD: $11.3 \%$, UC: $15.4 \%$ ), which was similar to that in the general population in Taiwan. Our patients had a mean age at diagnosis of 38.4 years, which was older than the HBV vaccination program. Being an endemic area of HBV infection, the prophylactic use of antiviral agents for patients with IBD with HBV infection is an important issue in Taiwan. Moreover, data on the seroprevalence of HCV infection in patients with IBD are limited and conflicting. Coban et al. reported that the seroprevalence of $\mathrm{HCV}$ infection in patients with IBD was $39.8 \%$ in CD cases and $41.6 \%$ in UC cases in China, 23.8\% in CD cases and 35.2\% in UC cases in South Korea, and lower in Europe [36]. We found no HCV infection in 158 patients with available data, compared with the previously reported incidence (3.28\%) in the general population in Taiwan [37].

A variety of serologic markers that are relevant to the diagnosis and treatment of IBD are emerging, including ANCAs, anti-Saccharomyces cerevisiae antibodies, antichitobioside carbohydrate antibodies, anti-laminaribioside carbohydrate antibodies, anti-mannobioside carbohydrate antibodies, and anti-outer membrane porins [38]. ANCAs, including p-ANCA and c-ANCA, are autoantibodies against neutrophil granules and monocyte lysosomes. Serum p-ANCA was first reported to be associated with $\mathrm{UC}$ and has been applied as a diagnostic tool for detecting IBD. Prideaux et al. conducted a review study and found that the prevalence of p-ANCA in patients with IBD was $6-38 \%$ in CD cases and $41-73 \%$ in UC cases [39]. Some studies have claimed that p-ANCA in UC was less prevalent in Asia than in Western countries [40,41]. To our knowledge thus far, there is no report on the ANCA prevalence in patients with IBD in Taiwan. In our present study, we identified the prevalence of p-ANCA as $22.2 \%$ in patients with UC, which was similar to that in a Korean report $(22.1 \%)$ but was lower than that in a report from China $(43.4 \%)[42,43]$. Moreover, we also identified that the prevalence of c-ANCA was $1.6 \%$ in patients with UC, which was lower than that in previous reports from Jeddah (3\%), Japan (39.2\%), and England (31\%) [44-46].

Concerning the treatment regimens, 5-ASAs and corticosteroids are highly preferred worldwide for the treatment of IBD. In the present study, 5-ASAs were the preferred regimen for the treatment of IBD, especially UC, which was consistent to that reported in other studies [47-49]. Concerning the use of immunomodulators, our present study demonstrated that immunomodulators were more often prescribed in patients with CD than in those with UC, which is comparable to the findings of other reports $[48,49]$. With respect to the use of biologic agents, $85.0 \%$ of our patients with $\mathrm{CD}$ and $42.7 \%$ of patients with UC received biologic treatments, which were much higher than those in previous Western and Asian reports (3-25\% vs. $8-11 \%$ in CD cases and $1-2 \%$ vs. $9 \%$ in UC cases) [40, 48-50]. Although many studies have demonstrated the efficacy of biologic agents for IBD, patients in some countries have to pay for biologic agents [51]. In Taiwan, we have a National Health Insurance system covering $>99 \%$ of the population, which partially pays for the use of biologic agents. Thus, patients with IBD can usually receive treatment with biologic agents after National Health Insurance verification in Taiwan. Therefore, our insurance system might be one of the reasons for the higher 
use of biologic agents. The bowel resection rates in patients with IBD vary among different regions and countries $[52,53]$. However, we found that the bowel resection rate in patients with IBD has decreased in some newer cohort studies. This might be attributed to medical advances, such as biologic agents and medical care, for patients with IBD. The bowel resection rate in our patients with $\mathrm{CD}$ was $38.8 \%$, which was similar to that reported in a Korean study (35.5\%) but was lower than that in European (38-61\%) and Japanese (55\%) reports after a 10-year follow-up [54-56]. Sato et al. reported that ileal-type CD had a significantly higher cumulative rate of surgery than the other types [55]. In our present study, $57.5 \%$ of patients with CD had the ileal type with poor disease behavior (stricturing and penetrating types were found in $>80 \%$ of the patients), which may be the reason for the higher bowel resection rate despite more advanced medical care such as the use of immunomodulators and biologic agents. We also found a colectomy rate of 3.6\% in patients with UC, which was lower than that reported in Korea (7.5\%), Japan (14.5\%), and Europe (2.8-24\%) $[50,56,57]$. AVN of the hips is associated with significant morbidity, potentially causing severe pain and debilitation. In the literature, there were large variations in the incidence of hip AVN in patients with IBD. Corticosteroids are a known risk factor for hip AVN [58]. Freeman and Freeman reported that the incidence rate of hip AVN in all patients with $\mathrm{CD}$ was less than $0.5 \%$, but it was $1 \%$ in male patients [59]. Recently, Rolston et al. reported that the incidence of hip AVN in patients with IBD was $2.1 \%$ [60]. They concluded that patients with IBD had a higher prevalence of AVN than patients without IBD. In our study, we reported a prevalence rate of hip AVN of $3.2 \%$ in all patients with IBD ( 3 CD cases and 3 UC cases), which was higher than that in previous reports. Our higher prevalence rate of hip AVN in patients with IBD may be due to the overuse and long-duration use of corticosteroids in Taiwan. Therefore, we suggest early tapering of corticosteroids and close surveillance for hip AVN in patients with IBD, especially those with prolonged use of corticosteroids.

\section{Conclusions}

In recent decades, the prevalence and incidence of IBD have been increasing but remain low in Taiwan. To date, only a few hospital-based studies have described the clinical characteristics and treatment outcomes of IBD in Taiwan. We conducted a retrospective hospital-based study in central Taiwan. We found that patients with IBD had a male predominance, lack of familial clustering, and lower prevalence of p-ANCA, EIMs, HCV infection, and CRCs. However, we observed a higher seroprevalence of HBsAg in patients with IBD than that reported in other Asian and Western countries. Thus, early detection of HBV infection and earlier antiviral prophylaxis for IBD are important issues in Taiwan. Moreover, our patients showed greater severity and higher rates of complications, use of biologic agents, hip AVN, and bowel resection than patients in other Asian and Western studies. Thus, we recommend early diagnosis and evaluation of complications, early use of immunomodulators and biologic agents, and avoidance of long-term corticosteroid use in patients with IBD. These precautions can help guide the management strategies to change the clinical course of patients with IBD in Taiwan. The limitations of our present study are its small sample size and retrospective nature. In the future, more prospective population-based studies at the national level are needed to identify the clinical characteristics contributing to the IBD prevalence and disease treatment outcomes in Taiwan.

\section{Data Availability}

The clinical data used to support the findings of this study are restricted by the institutional review board of the research ethics committee of China Medical University Hospital in order to protect the patients' privacy. Data are available from the corresponding author (Dr. Hsiang-Chun Lai, u9702451@cmu.edu.tw) for researchers who meet the criteria for access to confidential data.

\section{Conflicts of Interest}

The authors declare no conflicts of interest exist.

\section{Acknowledgments}

The authors thank the board members of the Big Data Center at China Medical University Hospital.

\section{References}

[1] J. Cosnes, C. Gower-Rousseau, P. Seksik, and A. Cortot, "Epidemiology and natural history of inflammatory bowel diseases," Gastroenterology, vol. 140, no. 6, pp. 1785-1794.e4, 2011.

[2] L. Prideaux, M. A. Kamm, P. P. de Cruz, F. K. L. Chan, and S. C. Ng, "Inflammatory bowel disease in Asia: a systematic review," Journal of Gastroenterology and Hepatology, vol. 27, no. 8, pp. 1266-1280, 2012.

[3] S. C. Ng, H. Y. Shi, N. Hamidi et al., "Worldwide incidence and prevalence of inflammatory bowel disease in the 21st century: a systematic review of population-based studies," The Lancet, vol. 390, no. 10114, pp. 2769-2778, 2018.

[4] J. J. Keller, J. Wang, Y. L. Hwang et al., "Increased risk of stroke among patients with Crohn's disease: a population-based matched cohort study," International Journal of Colorectal Disease, vol. 30, no. 5, pp. 645-653, 2015.

[5] S. C. Wei, M. J. Shieh, M. C. Chang, Y. T. Chang, C. Y. Wang, and J. M. Wong, "Long-term follow-up of ulcerative colitis in Taiwan," Journal of the Chinese Medical Association, vol. 75, no. 4, pp. 151-155, 2012.

[6] S. K. Yang, S. Yun, J. H. Kim et al., "Epidemiology of inflammatory bowel disease in the Songpa-Kangdong district, Seoul, Korea, 1986-2005: a KASID study," Inflammatory Bowel Diseases, vol. 14, no. 4, pp. 542-549, 2008.

[7] S. C. Wei, M. H. Lin, C. C. Tung et al., "A nationwide population-based study of the inflammatory bowel diseases between 1998 and 2008 in Taiwan," BMC Gastroenterology, vol. 13, no. 1, p. 166, 2013.

[8] S. C. Ng, G. G. Kaplan, W. Tang et al., "Population density and risk of inflammatory bowel disease: a prospective population- 
based study in 13 countries or regions in Asia-Pacific," The American Journal of Gastroenterology, vol. 114, no. 1, pp. 107-115, 2019.

[9] J. D. Betteridge, S. P. Armbruster, C. Maydonovitch, and G. R. Veerappan, "Inflammatory bowel disease prevalence by age, gender, race, and geographic location in the U.S. military health care population," Inflammatory Bowel Diseases, vol. 19, no. 7, pp. 1421-1427, 2013.

[10] T. A. Malik, "Inflammatory bowel disease: historical perspective, epidemiology, and risk factors," Surgical Clinics of North America, vol. 95, no. 6, pp. 1105-1122, 2015.

[11] G. C. Parkes, K. Whelan, and J. O. Lindsay, "Smoking in inflammatory bowel disease: impact on disease course and insights into the aetiology of its effect," Journal of Crohn's and Colitis, vol. 8, no. 8, pp. 717-725, 2014.

[12] B. Casetta, A. J. Videla, A. Bardach et al., "Association between cigarette smoking prevalence and income level: a systematic review and meta-analysis," Nicotine \& Tobacco Research, vol. 19, no. 12, pp. 1401-1407, 2017.

[13] T. Birrenbach and U. Bocker, "Inflammatory bowel disease and smoking: a review of epidemiology, pathophysiology, and therapeutic implications," Inflammatory Bowel Diseases, vol. 10, no. 6, pp. 848-859, 2004.

[14] Y. Zhang, R. Lin, J. Tao et al., "Electroacupuncture improves cognitive ability following cerebral ischemia reperfusion injury via CaM-CaMKIV-CREB signaling in the rat hippocampus," Experimental and Therapeutic Medicine, vol. 12, no. 2, pp. 777-782, 2016.

[15] K. T. Thia, E. V. Loftus, Jr, W. J. Sandborn, and S. K. Yang, "An update on the epidemiology of inflammatory bowel disease in Asia," The American Journal of Gastroenterology, vol. 103, no. 12, pp. 3167-3182, 2008.

[16] M. C. Harpsøe, K. T. Jørgensen, M. Frisch, and T. Jess, "Risk of inflammatory bowel disease according to self-rated health, pregnancy course, and pregnancy complications: a study within the Danish National Birth Cohort," PLoS One, vol. 8, no. 3, article e59698, 2013.

[17] G. Mantzouranis, E. Fafliora, M. Saridi et al., "Alcohol and narcotics use in inflammatory bowel disease," Annals of Gastroenterology, vol. 31, no. 6, pp. 649-658, 2018.

[18] S. L. Jowett, C. J. Seal, M. S. Pearce et al., "Influence of dietary factors on the clinical course of ulcerative colitis: a prospective cohort study," Gut, vol. 53, no. 10, pp. 1479-1484, 2004.

[19] G. R. Swanson, S. Sedghi, A. Farhadi, and A. Keshavarzian, "Pattern of alcohol consumption and its effect on gastrointestinal symptoms in inflammatory bowel disease," Alcohol, vol. 44, no. 3, pp. 223-228, 2010.

[20] A. M. El-Tawil, "Epidemiology and inflammatory bowel diseases," World Journal of Gastroenterology, vol. 19, no. 10, pp. 1505-1507, 2013.

[21] A. M. Bianco, V. Zanin, L. Monasta, S. Martelossi, A. Marcuzzi, and S. Crovella, "Family history in early-onset inflammatory bowel disease," Journal of Gastroenterology, vol. 48, no. 1, p. 144, 2013.

[22] R. E. Childers, S. Eluri, C. Vazquez, R. M. Weise, T. M. Bayless, and S. Hutfless, "Family history of inflammatory bowel disease among patients with ulcerative colitis: a systematic review and meta-analysis," Journal of Crohn's and Colitis, vol. 8, no. 11, pp. 1480-1497, 2014.

[23] S. K. Yang, "How does the epidemiology of inflammatory bowel disease differ between East and West? A Korean per- spective," Inflammatory Intestinal Diseases, vol. 2, no. 2, pp. 95-101, 2017.

[24] S. C. Wei, Y. H. Ni, H. I. Yang et al., "A hospital-based study of clinical and genetic features of Crohn's disease," Journal of the Formosan Medical Association, vol. 110, no. 9, pp. 600-606, 2011.

[25] D. A. Schwartz, E. V. Loftus Jr, W. J. Tremaine et al., "The natural history of fistulizing Crohn's disease in Olmsted County, Minnesota," Gastroenterology, vol. 122, no. 4, pp. 875-880, 2002.

[26] T. Kitahora, T. Utsunomiya, and A. Yokota, "Epidemiological study of ulcerative colitis in Japan: incidence and familial occurrence. The Epidemiology Group of the Research Committee of Inflammatory Bowel Disease in Japan," Journal of Gastroenterology, vol. 30, Supplement 8, pp. 5-8, 1995.

[27] M. Harbord, V. Annese, S. R. Vavricka et al., "The first European evidence-based consensus on extra-intestinal manifestations in inflammatory bowel disease," Journal of Crohn's and Colitis, vol. 10, no. 3, pp. 239-254, 2016.

[28] N. N. Andersen and T. Jess, "Has the risk of colorectal cancer in inflammatory bowel disease decreased?" World Journal of Gastroenterology, vol. 19, no. 43, pp. 7561-7568, 2013.

[29] J. A. Eaden, K. R. Abrams, and J. F. Mayberry, "The risk of colorectal cancer in ulcerative colitis: a meta-analysis," Gut, vol. 48, no. 4, pp. 526-535, 2001.

[30] A. Schweitzer, J. Horn, R. T. Mikolajczyk, G. Krause, and J. J. Ott, "Estimations of worldwide prevalence of chronic hepatitis B virus infection: a systematic review of data published between 1965 and 2013," The Lancet, vol. 386, no. 10003, pp. 1546-1555, 2015.

[31] M. L. Huang, X. T. Xu, J. Shen, Y. Q. Qiao, Z. H. Dai, and Z. H. Ran, "Prevalence and factors related to hepatitis B and C infection in inflammatory bowel disease patients in China: a retrospective study," Journal of Crohn's and Colitis, vol. 8, no. 4, pp. 282-287, 2014.

[32] Y. C. Chien, C. F. Jan, H. S. Kuo, and C. J. Chen, "Nationwide hepatitis B vaccination program in Taiwan: effectiveness in the 20 years after it was launched," Epidemiologic Reviews, vol. 28, no. 1, pp. 126-135, 2006.

[33] C. L. Chen, J. Y. Yang, S. F. Lin et al., "Slow decline of hepatitis B burden in general population: results from a populationbased survey and longitudinal follow-up study in Taiwan," Journal of Hepatology, vol. 63, no. 2, pp. 354-363, 2015.

[34] S. M. Chen, C. M. Kung, W. J. Yang, and H. L. Wang, "Efficacy of the nationwide hepatitis $B$ infant vaccination program in Taiwan," Journal of Clinical Virology, vol. 52, no. 1, pp. 11-16, 2011.

[35] Y. H. Ni, M. H. Chang, J. F. Wu, H. Y. Hsu, H. L. Chen, and D. S. Chen, "Minimization of hepatitis B infection by a 25-year universal vaccination program," Journal of Hepatology, vol. 57, no. 4, pp. 730-735, 2012.

[36] S. Coban, M. Kekilli, and S. Köklü, “Approach and management of patients with chronic hepatitis $\mathrm{B}$ and $\mathrm{C}$ during the course of inflammatory bowel disease," Inflammatory Bowel Diseases, vol. 20, no. 11, pp. 2142-2150, 2014.

[37] M. L. Yu, M. L. Yeh, P. C. Tsai et al., "Huge gap between clinical efficacy and community effectiveness in the treatment of chronic hepatitis C: a nationwide survey in Taiwan," Medicine, vol. 94, no. 13, article e690, 2015.

[38] G. Zhou, Y. Song, W. Yang et al., "ASCA, ANCA, ALCA and many more: are they useful in the diagnosis of inflammatory 
bowel disease?," Digestive Diseases, vol. 34, no. 1-2, pp. 90-97, 2016.

[39] L. Prideaux, P. de Cruz, S. C. Ng, and M. A. Kamm, "Serological antibodies in inflammatory bowel disease: a systematic review," Inflammatory Bowel Diseases, vol. 18, no. 7, pp. 1340-1355, 2012.

[40] L. Prideaux, M. A. Kamm, P. de Cruz, D. van Langenberg, S. C. $\mathrm{Ng}$, and I. Dotan, "Inflammatory bowel disease serology in Asia and the West," World Journal of Gastroenterology, vol. 19, no. 37, pp. 6207-6213, 2013.

[41] A. A. Elkadri, J. M. Stempak, T. D. Walters et al., "Serum antibodies associated with complex inflammatory bowel disease," Inflammatory Bowel Diseases, vol. 19, no. 7, pp. 1499-1505, 2013.

[42] F. Zhou, B. Xia, F. Wang et al., “The prevalence and diagnostic value of perinuclear antineutrophil cytoplasmic antibodies and anti-Saccharomyces cerevisiae antibodies in patients with inflammatory bowel disease in mainland China," Clinica Chimica Acta, vol. 411, no. 19-20, pp. 1461-1465, 2010.

[43] J. H. Lee, J. H. Cheon, E. S. Kim et al., "The prevalence and clinical significance of perinuclear anti-neutrophil cytoplasmic antibody in Korean patients with ulcerative colitis," Digestive Diseases and Sciences, vol. 55, no. 5, pp. 1406-1412, 2010.

[44] O. I. Saadah and J. A. Al-Mughales, "Pediatric inflammatory bowel disease with cytoplasmic staining of antineutrophil cytoplasmic antibodies," Clinical and Developmental Immunology, vol. 2013, Article ID 196012, 5 pages, 2013.

[45] H. Takedatsu, K. Mitsuyama, S. Fukunaga et al., "Diagnostic and clinical role of serum proteinase 3 antineutrophil cytoplasmic antibodies in inflammatory bowel disease," Journal of Gastroenterology and Hepatology, vol. 33, no. 9, pp. 1603-1607, 2018.

[46] M. Mahler, D. P. Bogdanos, P. Pavlidis et al., "PR3-ANCA: a promising biomarker for ulcerative colitis with extensive disease," Clinica Chimica Acta, vol. 424, pp. 267-273, 2013.

[47] J. J. Y. Sung, M. A. Kamm, and P. Marteau, “Asian perspectives in the management of inflammatory bowel disease: findings from a recent survey," Journal of Gastroenterology and Hepatology, vol. 25, no. 1, pp. 183-193, 2010.

[48] Z. Vegh, J. Burisch, N. Pedersen et al., "Incidence and initial disease course of inflammatory bowel diseases in 2011 in Europe and Australia: results of the 2011 ECCO-EpiCom inception cohort," Journal of Crohn's and Colitis, vol. 8, no. 11, pp. 1506-1515, 2014.

[49] S. Taleban, J. F. Colombel, M. J. Mohler, and M. J. Fain, "Inflammatory bowel disease and the elderly: a review," Journal of Crohn's and Colitis, vol. 9, no. 6, pp. 507-515, 2015.

[50] H. S. Lee, S. H. Park, S. K. Yang et al., "Long-term prognosis of ulcerative colitis and its temporal change between 1977 and 2013: a hospital-based cohort study from Korea," Journal of Crohn's and Colitis, vol. 9, no. 2, pp. 147-155, 2015.

[51] L. B. Cohen, R. M. Nanau, F. Delzor, and M. G. Neuman, "Biologic therapies in inflammatory bowel disease," Translational Research, vol. 163, no. 6, pp. 533-556, 2014.

[52] H. J. Kim, H. J. Hann, S. N. Hong et al., "Incidence and natural course of inflammatory bowel disease in Korea, 2006-2012: a nationwide population-based study," Inflammatory Bowel Diseases, vol. 21, no. 3, pp. 623-630, 2015.
[53] O. Icht, H. Yanai, Y. Ron et al., "Comparative study of two cohorts of newly diagnosed Crohn's disease demonstrates change in therapeutic strategies," Digestion, vol. 96, no. 3, pp. 135-141, 2017.

[54] S. H. Park, S. K. Yang, S. K. Park et al., "Long-term prognosis of Crohn's disease and its temporal change between 1981 and 2012: a hospital-based cohort study from Korea," Inflammatory Bowel Diseases, vol. 20, no. 3, pp. 488-494, 2014.

[55] Y. Sato, T. Matsui, Y. Yano et al., "Long-term course of Crohn's disease in Japan: incidence of complications, cumulative rate of initial surgery, and risk factors at diagnosis for initial surgery," Journal of Gastroenterology and Hepatology, vol. 30, no. 12, pp. 1713-1719, 2015.

[56] J. Burisch, T. Jess, M. Martinato, P. L. Lakatos, and ECCO -EpiCom, "The burden of inflammatory bowel disease in Europe," Journal of Crohn's and Colitis, vol. 7, no. 4, pp. 322-337, 2013.

[57] H. Shiga, S. Takagi, R. Inoue et al., "What determines the later clinical course of patients who do not undergo colectomy at the first attack? A Japanese cohort study on ulcerative colitis," Digestion, vol. 81, no. 2, pp. 104-112, 2010.

[58] D. Weldon, "The effects of corticosteroids on bone: osteonecrosis (avascular necrosis of the bone)," Annals of Allergy, Asthma \& Immunology, vol. 103, no. 2, pp. 91-98, 2009.

[59] H. J. Freeman and K. J. Freeman, "Prevalence rates and an evaluation of reported risk factors for osteonecrosis (avascular necrosis) in Crohn's disease," Canadian Journal of Gastroenterology, vol. 14, no. 2, pp. 138-143, 2000.

[60] V. S. Rolston, A. V. Patel, T. J. Learch et al., "Prevalence and associations of avascular necrosis of the hip in a large wellcharacterized cohort of patients with inflammatory bowel disease," JCR: Journal of Clinical Rheumatology, vol. 25, no. 1, pp. 45-49, 2019. 


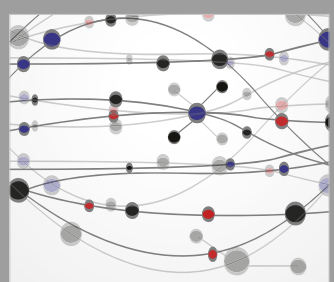

The Scientific World Journal
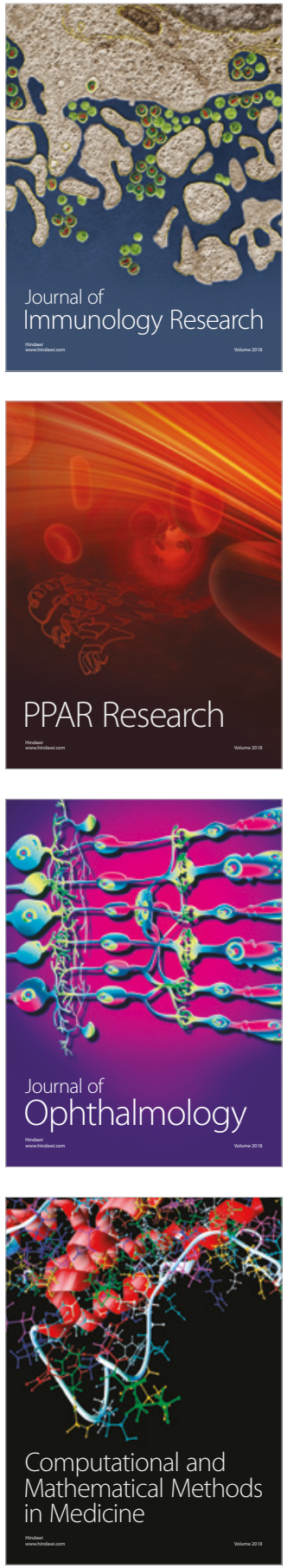

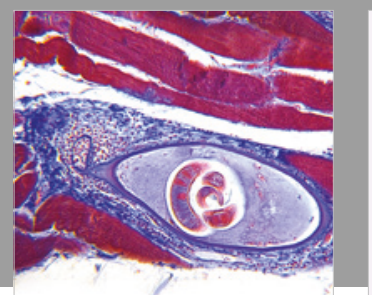

Gastroenterology Research and Practice

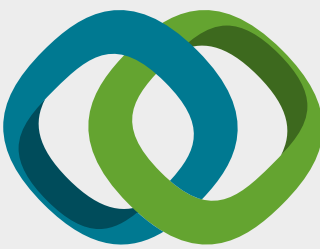

\section{Hindawi}

Submit your manuscripts at

www.hindawi.com
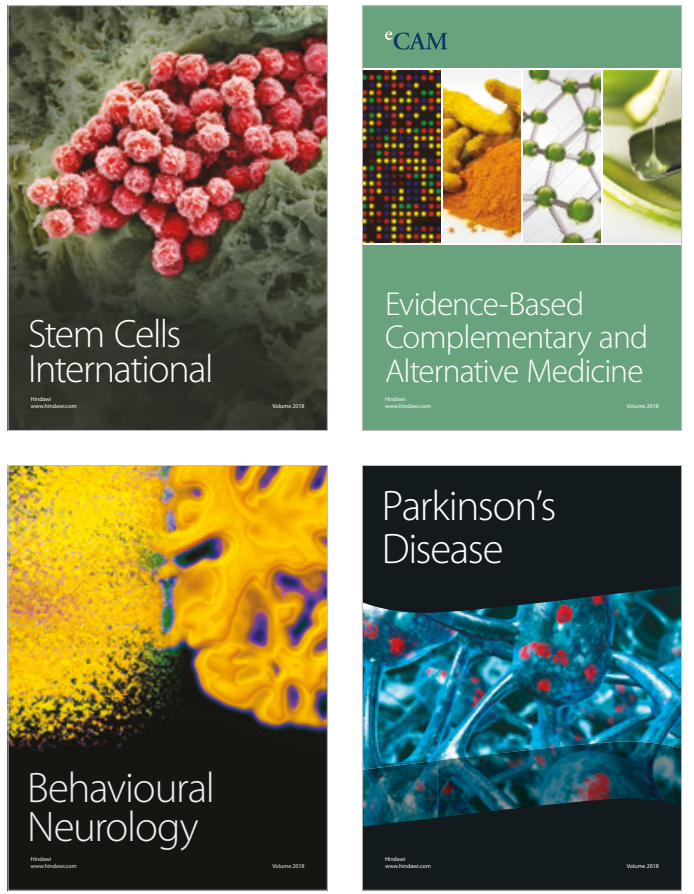

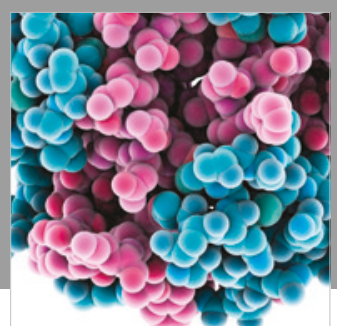

ournal of

Diabetes Research

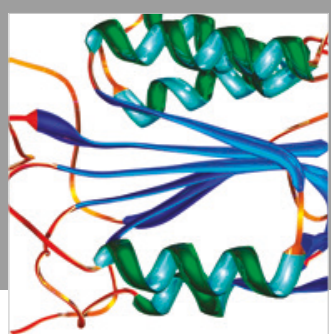

Disease Markers
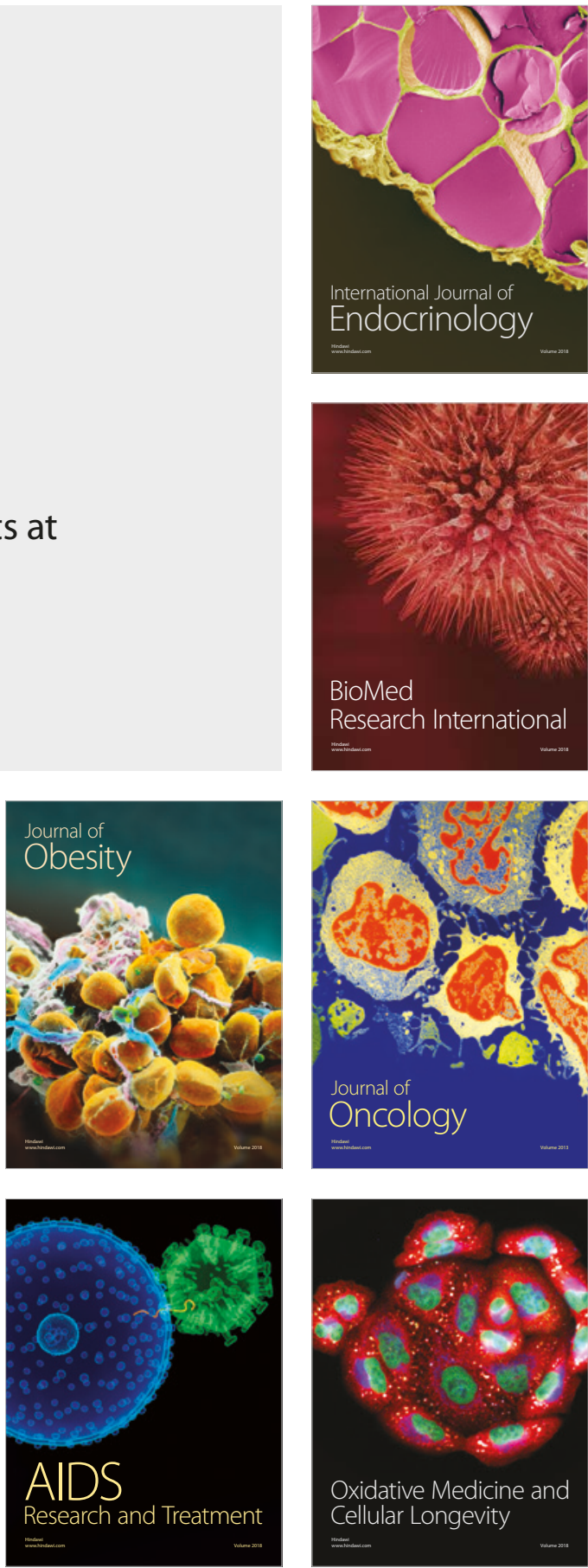